\title{
On the Behavior of Solutions of the System of Rational Difference Equations
}

$$
x_{n+1}=\frac{x_{n-1}}{y_{n} x_{n-1}-1}, \quad y_{n+1}=\frac{y_{n-1}}{x_{n} y_{n-1}-1}, \quad z_{n+1}=\frac{x_{n}}{y_{n} z_{n-1}}
$$

\author{
Abdullah Selçuk Kurbanlı ${ }^{1}$, Cengiz Çinar ${ }^{2}$, Mehmet Emre Erdoğan $^{3}$ \\ ${ }^{1}$ Mathematics Department, Education Faculty, Selçuk University, Konya, Turkey \\ ${ }^{2}$ Mathematics Department, Education Faculty, Gazi University, Ankara, Turkey \\ ${ }^{3}$ Huglu Vocational High School, Selçuk University, Konya, Turkey \\ E-mail:agurban@selcuk.edu.tr,ccinar25@yahoo.com,m_emre448@hotmail.com \\ Received April 29, 2011; revised May 16, 2011; accepted May 24, 2011
}

\section{Abstract}

In this paper, we investigate the solutions of the system of difference equations $x_{n+1}=\frac{x_{n-1}}{y_{n} x_{n-1}-1}$, $y_{n+1}=\frac{y_{n-1}}{x_{n} y_{n-1}-1}, \quad z_{n+1}=\frac{x_{n}}{y_{n} z_{n-1}}$ where $x_{0}, x_{-1}, y_{0}, y_{-1}, z_{0}, z_{-1} \in \mathfrak{R}$.

Keywords: Difference Equation, Difference Equation Systems, Solutions

\section{Introduction}

Recently, there has been great interest in studying difference equation systems. One of the reasons for this is a necessity for some techniques which can be used in investigating equations arising in mathematical models describing real life situations in population biology, economic, probability theory, genetics, psychology etc. Although difference equations are very simple in form, it is extremely difficult to understand throughly the global behavior of their solutions; for example, see Refs. [1-28].

In this paper, we investigated the periodicity of the solutions of the difference equation system

$$
x_{n+1}=\frac{x_{n-1}}{y_{n} x_{n-1}-1}, \quad y_{n+1}=\frac{y_{n-1}}{x_{n} y_{n-1}-1}, \quad z_{n+1}=\frac{x_{n}}{y_{n} z_{n-1}}
$$

where the initial conditions are arbitrary real numbers.

\section{Main Results}

\section{Theorem 1. Let}

$y_{0}=a, y_{-1}=b, x_{0}=c, x_{-1}=d, z_{0}=e, z_{-1}=f$ be arbitrary real numbers and let $\left\{x_{n}, y_{n}, z_{n}\right\}$ be a solutions of the system (1.1). Also, assume that $a d \neq 1$ and $c b \neq 1$, $a \neq 0, b \neq 0, e \neq 0$ and $f \neq 0$. Then all solutions of (1.1) are

$$
\begin{aligned}
& x_{n}=\left\{\begin{array}{l}
\frac{d}{(a d-1)^{n}}, n \text {-odd } \\
c(c b-1)^{n}, n-\text { even }
\end{array}\right. \\
& y_{n}=\left\{\begin{array}{l}
\frac{b}{(c b-1)^{n}}, n \text {-odd } \\
a(a d-1)^{n}, n-\text { even }
\end{array}\right. \\
& z_{n}=\left\{\begin{array}{l}
\frac{c(c b-1)^{n-1}}{a f(a d-1)^{n-1}}, \quad n=4 k-3, k=1,2,3, \cdots \\
\frac{d(c b-1)^{n-1}}{b e(a d-1)^{n-1}}, \quad n=4 k-2, k=1,2,3, \cdots \\
\frac{f(c b-1)^{n-1}}{(a d-1)^{n-1}}, \quad n=4 k-1, k=1,2,3, \cdots \\
\frac{e(c b-1)^{n-1}}{(a d-1)^{n-1}}, \quad n=4 k \quad, k=1,2,3, \cdots
\end{array}\right.
\end{aligned}
$$


Proof: For $n=1,2,3$, we have

$$
\begin{aligned}
& x_{1}=\frac{x_{-1}}{y_{0} x_{-1}-1}=\frac{d}{a d-1} \\
& y_{1}=\frac{y_{-1}}{x_{0} y_{-1}-1}=\frac{b}{c b-1} \\
& z_{1}=\frac{x_{0}}{y_{0} z_{-1}}=\frac{c}{a f} \text {, } \\
& x_{2}=\frac{x_{01}}{y_{1} x_{0}-1}=\frac{c}{\frac{b}{c b-1} c-1}=c(c b-1) \\
& y_{2}=\frac{y_{0}}{x_{1} y_{0}-1}=\frac{a}{\frac{d}{a d-1} a-1}=a(a d-1) \\
& z_{2}=\frac{x_{1}}{y_{1} z_{0}}=\frac{\frac{d}{a d-1}}{\frac{b}{c b-1} e}=\frac{d(c b-1)}{b e(a d-1)} \\
& x_{3}=\frac{x_{1}}{y_{2} x_{1}-1}=\frac{\frac{d}{a d-1}}{a(a d-1) \frac{d}{a d-1}-1}=\frac{d}{(a d-1)^{2}} \text {, } \\
& y_{3}=\frac{y_{1}}{x_{2} y_{1}-1}=\frac{\frac{b}{c b-1}}{c(c b-1) \frac{b}{c b-1}-1}=\frac{b}{(c b-1)^{2}} \\
& z_{3}=\frac{x_{2}}{y_{2} z_{1}}=\frac{c(c b-1)}{a(a d-1) \frac{c}{a f}}=\frac{f(c b-1)}{(a d-1)} \text {, } \\
& x_{4}=\frac{x_{2}}{y_{3} x_{2}-1}=\frac{c(c b-1)}{\frac{b}{(c b-1)^{2}} c(c b-1)-1}=\frac{c(c b-1)}{\frac{c b}{c b-1}-1} \\
& =c(c b-1)^{2} \\
& y_{4}=\frac{y_{2}}{x_{3} y_{2}-1}=\frac{a(a d-1)}{\frac{d}{(a d-1)^{2}} a(a d-1)-1}=\frac{a(a d-1)}{\frac{a d}{a d-1}-1} \\
& =a(a d-1)^{2} \\
& z_{4}=\frac{x_{3}}{y_{3} z_{2}}=\frac{\frac{d}{(a d-1)^{2}}}{\frac{b}{(c b-1)^{2}} \frac{d(c b-1)}{b e(a d-1)}}=\frac{e(c b-1)}{(a d-1)}
\end{aligned}
$$

for $n=k$ assume that

$$
\begin{aligned}
& x_{2 k-1}=\frac{x_{2 k-3}}{y_{2 k-2} x_{2 k-3}-1}=\frac{d}{(a d-1)^{k}} \\
& x_{2 k}=\frac{x_{2 k-2}}{y_{2 k-1} x_{2 k-2}-1}=c(c b-1)^{k} \\
& y_{2 k-1}=\frac{y_{2 k-3}}{x_{2 k-2} y_{2 k-3}-1}=\frac{b}{(c b-1)^{k}} \\
& y_{2 k}=\frac{y_{2 k-2}}{x_{2 k-1} y_{2 k-2}-1}=a(a d-1)^{k}
\end{aligned}
$$

and

$$
\begin{gathered}
z_{4 k-3}=\frac{x_{4 k-4}}{y_{4 k-4} z_{4 k-5}}=\frac{c(c b-1)^{k-1}}{a f(a d-1)^{k-1}} \\
z_{4 k-2}=\frac{x_{4 k-3}}{y_{4 k-3} z_{4 k-4}}=\frac{d(c b-1)^{k}}{b e(a d-1)^{k}} \\
z_{4 k-1}=\frac{x_{4 k-2}}{y_{4 k-2} z_{4 k-3}}=\frac{f(c b-1)^{k}}{(a d-1)^{k}} \\
z_{4 k}=\frac{x_{4 k-1}}{y_{4 k-1} z_{4 k-2}}=\frac{e(c b-1)^{k}}{(a d-1)^{k}}
\end{gathered}
$$

are true. Then $n=k+1$ we will show that (1.2), (1.3) and (1.4) are true. From (1.1), we have

$$
\begin{aligned}
x_{2 k+1} & =\frac{x_{2 k-1}}{y_{2 k} x_{2 k-1}-1}=\frac{\frac{d}{(a d-1)^{k}}}{a(a d-1)^{k} \frac{d}{(a d-1)^{k}}-1} \\
& =\frac{d}{(a d-1)^{k+1}} \\
y_{2 k+1} & =\frac{y_{2 k-1}}{x_{2 k} y_{2 k-1}-1}=\frac{\frac{b}{(c b-1)^{k}}}{c(c b-1)^{k} \frac{b}{(c b-1)^{k}}-1} . \\
& =\frac{b}{(c b-1)^{k+1}}
\end{aligned}
$$

Also, similarly from (1.1), we have

$$
\begin{aligned}
& z_{4 k+1}=\frac{x_{4 k}}{y_{4 k} z_{4 k-1}}=\frac{c(c b-1)^{2 k}}{a(a d-1)^{2 k} \frac{f(c b-1)^{k}}{(a d-1)^{k}}}=\frac{c(c b-1)^{k}}{a f(a d-1)^{k}} \\
& Z_{4 k+2}=\frac{x_{4 k+1}}{y_{4 k+1} z_{4 k}}=\frac{\frac{d}{(a d-1)^{2 k+1}}}{\frac{b}{(c b-1)^{2 k+1}} \frac{e(c b-1)^{k}}{(a d-1)^{k}}}=\frac{d(c b-1)^{k+1}}{b e(a d-1)^{k+1}}
\end{aligned}
$$


Also, we have

$$
\begin{aligned}
x_{2 k+2} & =\frac{x_{2 k}}{y_{2 k+1} x_{2 k-1}}=\frac{c(c b-1)^{k}}{\frac{b}{(c b-1)^{k+1}} c(c b-1)^{k}-1}=\frac{c(c b-1)^{k}}{\frac{b}{c b-1} c-1} \\
& =c(c b-1)^{k+1} \\
y_{2 k+2} & =\frac{y_{2 k}}{x_{2 k+1} y_{2 k}-1}=\frac{a(a d-1)^{k}}{\frac{d}{(a d-1)^{k+1}} a(a d-1)^{k}-1}=\frac{a(a d-1)^{k}}{\frac{d}{a d-1} a-1} \\
& =a(a d-1)^{k+1}
\end{aligned}
$$

and

$$
\begin{aligned}
z_{4 k+3} & =\frac{x_{4 k+2}}{y_{4 k+2} z_{4 k+1}}=\frac{c(c b-1)^{2 k+1}}{a(a d-1)^{2 k+1} \frac{c(c b-1)^{k}}{a f(a d-1)^{k}}} \\
& =\frac{f(c b-1)^{k+1}}{(a d-1)^{k+1}} \\
Z_{4 k+4} & =\frac{x_{4 k+3}}{y_{4 k+3} z_{4 k+2}}=\frac{\frac{d}{(a d-1)^{2 k+2}}}{\frac{d(c b-1)^{k+1}}{(c b-1)^{2 k+2}} \frac{b e(a d-1)^{k+1}}{(a d-1)^{k+1}}} \\
& =\frac{e(c b-1)^{k+1}}{(a d}
\end{aligned}
$$

Corollary 1. Let $a, b, c, d, e, f$ be arbitrary real numbers and let $\left\{x_{n}, y_{n}, z_{n}\right\}$ be a solution of the system (1.1). If $0<a, b, c, d, e, f<1$ then we have

$$
\begin{gathered}
\lim _{n \rightarrow \infty} x_{2 n-1}=\lim _{n \rightarrow \infty} y_{2 n-1}=\infty \\
\lim _{n \rightarrow \infty} z_{4 n-3}=\left\{\begin{array}{l}
\infty, c b<a d \\
\frac{c}{a f}, c b=a d
\end{array}, \lim _{n \rightarrow \infty} z_{4 n-1}= \begin{cases}0, & c b<a d \\
\infty, & c b>a d \\
f, & c b=a d\end{cases} \right.
\end{gathered}
$$

and

$$
\begin{gathered}
\lim _{n \rightarrow \infty} x_{2 n}=\lim _{n \rightarrow \infty} y_{2 n}=0 \\
\lim _{n \rightarrow \infty} z_{4 n-2}=\left\{\begin{array}{rl}
0, & c b<a d \\
\infty, & c b>a d, \lim _{n \rightarrow \infty} z_{4 n} \\
\frac{d}{b e}, & c b=a d
\end{array}= \begin{cases}0, & c b<a d \\
\infty, & c b>a d \\
e, & c b=a d\end{cases} \right.
\end{gathered}
$$

Proof. From $0<a, b, c, d<1$ we have

$$
0<\text { ad }<1 \Rightarrow-1<\text { ad }-1<0
$$

and

$$
0<c b<1 \Rightarrow-1<c b-1<0
$$

Hence, we obtain

$$
\begin{aligned}
\lim _{n \rightarrow \infty} x_{2 n-1} & =\lim _{n \rightarrow \infty} \frac{d}{(a d-1)^{n}}=d \lim _{n \rightarrow \infty} \frac{1}{(a d-1)^{n}} \\
& =d . \infty= \begin{cases}-\infty, & n \text {-odd } \\
+\infty, & n-\text { even }\end{cases} \\
\lim _{n \rightarrow \infty} y_{2 n-1} & =\lim _{n \rightarrow \infty} \frac{d}{(c b-1)^{n}}=b \lim _{n \rightarrow \infty} \frac{1}{(c b-1)^{n}} \\
& =b . \infty= \begin{cases}-\infty, & n-\text { odd } \\
+\infty, & n-\text { even }\end{cases}
\end{aligned}
$$

and

$$
\begin{aligned}
\lim _{n \rightarrow \infty} z_{4 n-3}= & \lim _{n \rightarrow \infty} \frac{c(c b-1)^{n-1}}{a f(a d-1)^{n-1}}=\lim _{n \rightarrow \infty} \frac{c}{a f}\left(\frac{c b-1}{a d-1}\right)^{n-1} \\
& =\left\{\begin{array}{l}
a d>c b \Rightarrow a d-1>c b-1 \Rightarrow \frac{c b-1}{a d-1}<1 \\
a d<c b \Rightarrow a d-1<c b-1 \Rightarrow \frac{c b-1}{a d-1}>1 \\
a d=c b \Rightarrow a d-1=c b-1 \Rightarrow \frac{c b-1}{a d-1}=1
\end{array}\right\} \\
= & \begin{cases}\infty, \quad c b>a d \\
\frac{c}{a f}, \quad c b=a d\end{cases}
\end{aligned}
$$

$$
\begin{aligned}
\lim _{n \rightarrow \infty} z_{4 n-1}= & \lim _{n \rightarrow \infty} \frac{f(c b-1)^{n}}{(a d-1)^{n}}=\lim _{n \rightarrow \infty} f\left(\frac{a b-1}{a d-1}\right)^{n} \\
& \left\{\begin{array}{l}
a d>c b \Rightarrow a d-1>c b-1 \Rightarrow \frac{c b-1}{a d-1}<1 \\
a d<c b \Rightarrow a d-1<c b-1 \Rightarrow \frac{c b-1}{a d-1}>1 \\
a d=c b \Rightarrow a d-1=c b-1 \Rightarrow \frac{c b-1}{a d-1}=1
\end{array}\right\} \\
= & \begin{cases}0, \quad c b<a d \\
\infty, \quad c b>a d \\
f, \quad c b=a d\end{cases}
\end{aligned}
$$

Similarly, we have

$$
\lim _{n \rightarrow \infty} x_{2 n}=\lim _{n \rightarrow \infty} c(c d-1)^{n}=c \lim _{n \rightarrow \infty}(c d-1)^{n}=c \cdot 0=0
$$




$$
\lim _{n \rightarrow \infty} y_{2 n}=\lim _{n \rightarrow \infty} c(a f-1)^{n}=a \lim _{n \rightarrow \infty}(a f-1)^{n}=a \cdot 0=0
$$

and

$$
\begin{aligned}
& \lim _{n \rightarrow \infty} z_{4 n-2}= \lim _{n \rightarrow \infty} \frac{d(c b-1)^{n}}{b e(a d-1)^{n}}=\lim _{n \rightarrow \infty} \frac{d}{b e}\left(\frac{c b-1}{a d-1}\right)^{n} \\
& a d>c b \Rightarrow a d-1>c b-1 \Rightarrow \frac{c b-1}{a d-1}<1 \\
&=\left\{\begin{array}{l}
a d<c b \Rightarrow a d-1<c b-1 \Rightarrow \frac{c b-1}{a d-1}>1 \\
a d=c b \Rightarrow a d-1=c b-1 \Rightarrow \frac{c b-1}{a d-1}=1
\end{array}\right\} \\
&= \begin{cases}0, \quad c b<a d \\
\frac{d}{b e}, \quad c b=a d\end{cases}
\end{aligned}
$$$$
\lim _{n \rightarrow \infty} z_{4 n}=\lim _{n \rightarrow \infty} \frac{e(c b-1)^{n}}{(a d-1)^{n}}=\lim _{n \rightarrow \infty} e\left(\frac{c b-1}{a d-1}\right)^{n}
$$$$
=\left\{\begin{array}{c}
a d>c b \Rightarrow a d-1>c b-1 \Rightarrow \frac{c b-1}{a d-1}<1 \\
a d<c b \Rightarrow a d-1<c b-1 \Rightarrow \frac{c b-1}{a d-1}>1 \\
a d=c b \Rightarrow a d-1=c b-1 \Rightarrow \frac{c b-1}{a d-1}=1
\end{array}\right\}
$$$$
= \begin{cases}0, & c b<a d \\ \infty, & c b>a d \\ e, & c b=a d\end{cases}
$$

Corollary 2. Let $a, b, c, d, e, f$ be arbitrary real numbers and let $\left\{x_{n}, y_{n}, z_{n}\right\}$ be a solution of the system (1.1). If $1<a d, c b<2, e \neq 0$ and $f \neq 0$ then we have

$$
\begin{aligned}
& \lim _{n \rightarrow \infty} x_{2 n-1}=\lim _{n \rightarrow \infty} y_{2 n-1}=\infty \\
& \lim _{n \rightarrow \infty} z_{4 n-3}= \begin{cases}0, & c b<a d \\
\infty, & c b>a d \\
\frac{c}{a f}, & c b=a d\end{cases} \\
& \lim _{n \rightarrow \infty} z_{4 n-1}= \begin{cases}0, & c b<a d \\
\infty, & c b>a d \\
f, & c b=a d\end{cases}
\end{aligned}
$$

and

$$
\begin{aligned}
& \lim _{n \rightarrow \infty} x_{2 n}=\lim _{n \rightarrow \infty} y_{2 n}=0 \\
& \lim _{n \rightarrow \infty} z_{4 n-2}=\left\{\begin{array}{cc}
0, & c b<a d \\
\infty, & c b>a d \\
\frac{d}{b e}, & c b=a d
\end{array}\right. \\
& \lim _{n \rightarrow \infty} z_{4 n}= \begin{cases}0, & c b<a d \\
\infty, & c b>a d \\
e, & c b=a d\end{cases}
\end{aligned}
$$

Proof. From $1<a d<2 \Rightarrow 0<a d-1<1$ we have

$$
\lim _{n \rightarrow \infty}(a d-1)^{n}=0
$$

from $1<c b<2 \Rightarrow 0<c b-1<1$ we have

$$
\lim _{n \rightarrow \infty}(c b-1)^{n}=0
$$

Hence, we have

$$
\begin{aligned}
\lim _{n \rightarrow \infty} x_{2 n-1} & =\lim _{n \rightarrow \infty} \frac{d}{(a d-1)^{n}}=d \lim _{n \rightarrow \infty} \frac{1}{(a d-1)^{n}}=d \cdot \infty \\
& = \begin{cases}-\infty, & d<0 \\
+\infty, & d>0\end{cases} \\
\lim _{n \rightarrow \infty} y_{2 n-1} & =\lim _{n \rightarrow \infty} \frac{b}{(c b-1)^{n}}=b \lim _{n \rightarrow \infty} \frac{1}{(c b-1)^{n}}=b \cdot \infty \\
& = \begin{cases}-\infty, & b<0 \\
+\infty, & b>0\end{cases}
\end{aligned}
$$

and

$$
\begin{aligned}
\lim _{n \rightarrow \infty} z_{4 n-3}= & \lim _{n \rightarrow \infty} \frac{c(c b-1)^{n-1}}{a f(a d-1)^{n-1}}=\lim _{n \rightarrow \infty} \frac{c}{a f}\left(\frac{c b-1}{a d-1}\right)^{n-1} \\
& =\left\{\begin{array}{l}
a d>c b \Rightarrow a d-1>c b-1 \Rightarrow \frac{c b-1}{a d-1}<1 \\
a d<c b \Rightarrow a d-1<c b-1 \Rightarrow \frac{c b-1}{a d-1}>1 \\
a d=c b \Rightarrow a d-1=c b-1 \Rightarrow \frac{c b-1}{a d-1}=1
\end{array}\right\} \\
= & \left\{\begin{array}{l}
\infty, \quad c b>a d \\
\frac{c}{a f}, \quad c b=a d
\end{array}\right.
\end{aligned}
$$




$$
\begin{aligned}
\lim _{n \rightarrow \infty} z_{4 n-1}= & \lim _{n \rightarrow \infty} \frac{f(c b-1)^{n}}{(a d-1)^{n}}=\lim _{n \rightarrow \infty} f\left(\frac{c b-1}{a d-1}\right)^{n} \\
& =\left\{\begin{array}{l}
a d>c b \Rightarrow a d-1>c b-1 \Rightarrow \frac{c b-1}{a d-1}<1 \\
a d<c b \Rightarrow a d-1<c b-1 \Rightarrow \frac{c b-1}{a d-1}>1 \\
a d=c b \Rightarrow a d-1=c b-1 \Rightarrow \frac{c b-1}{a d-1}=1
\end{array}\right\} \\
= & \begin{cases}0, \quad c b<a d \\
\infty, \quad c b>a d \\
f, \quad c b=a d\end{cases}
\end{aligned}
$$

Similarly, we have

$$
\begin{aligned}
& \lim _{n \rightarrow \infty} x_{2 n}=\lim _{n \rightarrow \infty} c(c b-1)^{n}=c \lim _{n \rightarrow \infty}(c b-1)^{n}=c \cdot 0=0 \\
& \lim _{n \rightarrow \infty} y_{2 n}=\lim _{n \rightarrow \infty} a(a d-1)^{n}=a \lim _{n \rightarrow \infty}(a d-1)^{n}=a \cdot 0=0
\end{aligned}
$$

and

$$
\begin{aligned}
& \lim _{n \rightarrow \infty} z_{4 n-2}=\lim _{n \rightarrow \infty} \frac{d(c b-1)^{n}}{b e(a d-1)^{n}}=\lim _{n \rightarrow \infty} \frac{d}{b e}\left(\frac{c b-1}{a d-1}\right)^{n} \\
& =\left\{\begin{array}{l}
a d>c b \Rightarrow a d-1>c b-1 \Rightarrow \frac{c b-1}{a d-1}<1 \\
a d<c b \Rightarrow a d-1<c b-1 \Rightarrow \frac{c b-1}{a d-1}>1 \\
a d=c b \Rightarrow a d-1=c b-1 \Rightarrow \frac{c b-1}{a d-1}=1
\end{array}\right\} \\
& =\left\{\begin{array}{cc}
0, & c b<a d \\
\infty, & c b>a d \\
\frac{d}{b e}, & c b=a d
\end{array}\right.
\end{aligned}
$$$$
\lim _{n \rightarrow \infty} z_{4 n}=\lim _{n \rightarrow \infty} \frac{e(c b-1)^{n}}{(a d-1)^{n}}=\lim _{n \rightarrow \infty} e\left(\frac{c b-1}{a d-1}\right)^{n}
$$$$
=\left\{\begin{array}{l}
a d>c b \Rightarrow a d-1>c b-1 \Rightarrow \frac{c b-1}{a d-1}<1 \\
a d<c b \Rightarrow a d-1<c b-1 \Rightarrow \frac{c b-1}{a d-1}>1 \\
a d=c b \Rightarrow a d-1=c b-1 \Rightarrow \frac{c b-1}{a d-1}=1
\end{array}\right\}
$$$$
= \begin{cases}0, & c b<a d \\ \infty, & c b>a d \\ e, & c b=a d\end{cases}
$$

Corollary 3. Let $\left\{x_{n}, y_{n}, z_{n}\right\}$ be the solutions of (1.1). If $c b, a d \in(-\infty, 0), e \neq 0$ and $f \neq 0$ then

$$
\begin{aligned}
& \lim _{n \rightarrow \infty} x_{2 n-1}=\lim _{n \rightarrow \infty} y_{2 n-1}=0 \\
& \lim _{n \rightarrow \infty} z_{4 n-3}=\left\{\begin{array}{cc}
0, & c b<a d \\
\infty, & c b>a d \\
\frac{c}{a f}, & c b=a d
\end{array}\right. \\
& \lim _{n \rightarrow \infty} z_{4 n-1}= \begin{cases}0, & c b<a d \\
\infty, & c b>a d \\
f, & c b=a d\end{cases}
\end{aligned}
$$

and

$$
\lim _{n \rightarrow \infty} x_{2 n}=\lim y_{2 n}=\infty
$$

$$
\lim _{n \rightarrow \infty} z_{4 n-2}=\left\{\begin{array}{cc}
0, & c b<a d \\
\infty, & c b>a d \\
\frac{d}{b e}, & c b=a d
\end{array}\right.
$$$$
\lim _{n \rightarrow \infty} z_{4 n}= \begin{cases}0, & c b<a d \\ \infty, & c b>a d \\ e, & c b=a d\end{cases}
$$

Proof. From $-\infty<c b<0 \Rightarrow-\infty<c b-1<-1$ and $-\infty<a d<0 \Rightarrow-\infty<a d-1<-1$ we have

$$
\lim _{n \rightarrow \infty}(c b-1)^{n}= \begin{cases}-\infty, & n-\text { odd } \\ +\infty, & n \text {-even }\end{cases}
$$

and

$$
\lim _{n \rightarrow \infty}(\text { ad }-1)^{n}= \begin{cases}-\infty, & n-\text { odd } \\ +\infty, & n-\text { even }\end{cases}
$$

Hence, we have

$$
\begin{aligned}
& \lim _{n \rightarrow \infty} x_{2 n-1}=\lim _{n \rightarrow \infty} \frac{d}{(a d-1)^{n}}=d \lim _{n \rightarrow \infty} \frac{1}{(a d-1)^{n}}=d \cdot 0=0 \\
& \lim _{n \rightarrow \infty} y_{2 n-1}=\lim _{n \rightarrow \infty} \frac{b}{(c b-1)^{n}}=b \lim _{n \rightarrow \infty} \frac{1}{(c b-1)^{n}}=b \cdot 0=0
\end{aligned}
$$

and 


$$
\begin{aligned}
& \lim _{n \rightarrow \infty} z_{4 n-3}=\lim _{n \rightarrow \infty} \frac{c(c b-1)^{n-1}}{a f(a d-1)^{n-1}}=\lim _{n \rightarrow \infty} \frac{c}{a f}\left(\frac{c b-1}{a d-1}\right)^{n-1} \\
& =\left\{\begin{array}{c}
a d>c b \Rightarrow a d-1>c b-1 \Rightarrow \frac{c b-1}{a d-1}<1 \\
a d<c b \Rightarrow a d-1<c b-1 \Rightarrow \frac{c b-1}{a d-1}>1 \\
a d=c b \Rightarrow a d-1=c b-1 \Rightarrow \frac{c b-1}{a d-1}=1
\end{array}\right\} \\
& =\left\{\begin{array}{cc}
0, & c b<a d \\
\infty, & c b>a d \\
\frac{c}{a f}, & c b=a d
\end{array}\right. \\
& \lim _{n \rightarrow \infty} z_{4 n-1}=\lim _{n \rightarrow \infty} \frac{f(c b-1)^{n}}{(a d-1)^{n}}=\lim _{n \rightarrow \infty} f\left(\frac{c b-1}{a d-1}\right)^{n} \\
& =\left\{\begin{array}{l}
a d>c b \Rightarrow a d-1>c b-1 \Rightarrow \frac{c b-1}{a d-1}<1 \\
a d<c b \Rightarrow a d-1<c b-1 \Rightarrow \frac{c b-1}{a d-1}>1 \\
a d=c b \Rightarrow a d-1=c b-1 \Rightarrow \frac{c b-1}{a d-1}=1
\end{array}\right\} \\
& = \begin{cases}0, & c b<a d \\
\infty, & c b>a d \\
f, & c b=a d\end{cases}
\end{aligned}
$$

Similarly, we have

$$
\begin{aligned}
\lim _{n \rightarrow \infty} x_{2 n}= & \lim _{n \rightarrow \infty} c(c b-1)^{n}=c \lim _{n \rightarrow \infty}(c b-1)^{n}=c \cdot \infty \\
& =\left\{\begin{array}{llll}
-\infty, & c>0 & \text { and } & n-\text { odd } \\
+\infty, & c<0 & \text { and } & n-\text { odd } \\
+\infty, & c>0 & \text { and } & n-\text { even } \\
-\infty, & c<0 & \text { and } & n-\text { even }
\end{array}\right.
\end{aligned}
$$

$$
\begin{aligned}
\lim _{n \rightarrow \infty} y_{2 n}= & \lim _{n \rightarrow \infty} a(\text { ad }-1)^{n}=a \lim _{n \rightarrow \infty}(\text { ad }-1)^{n}=a \cdot \infty \\
= & \left\{\begin{array}{llll}
-\infty, & a>0 & \text { and } & n \text {-odd } \\
+\infty, & a<0 & \text { and } & n \text {-odd } \\
+\infty, & a>0 & \text { and } & n \text {-even } \\
-\infty, & a<0 & \text { and } & n \text {-even }
\end{array}\right.
\end{aligned}
$$

and

$$
\begin{aligned}
& \lim _{n \rightarrow \infty} z_{4 n-2}= \lim _{n \rightarrow \infty} \frac{d(c b-1)^{n}}{b e(a d-1)^{n}}=\lim _{n \rightarrow \infty} \frac{d}{b e}\left(\frac{c b-1}{a d-1}\right)^{n} \\
& a d>c b \Rightarrow a d-1>c b-1 \Rightarrow \frac{c b-1}{a d-1}<1 \\
&=\left\{\begin{array}{l}
a d<c b \Rightarrow a d-1<c b-1 \Rightarrow \frac{c b-1}{a d-1}>1 \\
a d=c b \Rightarrow a d-1=c b-1 \Rightarrow \frac{c b-1}{a d-1}=1
\end{array}\right\} \\
&= \begin{cases}0, \quad c b>a d \\
\frac{d}{b e}, \quad c b=a d\end{cases}
\end{aligned}
$$$$
\lim _{n \rightarrow \infty} z_{4 n}=\lim _{n \rightarrow \infty} \frac{e(c b-1)^{n}}{(a d-1)^{n}}=\lim _{n \rightarrow \infty} e\left(\frac{c b-1}{a d-1}\right)^{n}
$$$$
=\left\{\begin{array}{c}
a d>c b \Rightarrow a d-1>c b-1 \Rightarrow \frac{c b-1}{a d-1}<1 \\
a d<c b \Rightarrow a d-1<c b-1 \Rightarrow \frac{c b-1}{a d-1}>1 \\
a d=c b \Rightarrow a d-1=c b-1 \Rightarrow \frac{c b-1}{a d-1}=1
\end{array}\right\}
$$$$
= \begin{cases}0, & c b<a d \\ \infty, & c b>a d \\ e, & c b=a d\end{cases}
$$

Corollary 4. Let $\left\{x_{n}, y_{n}\right\}$ be the solutions of (1.1). If $a, b, c, d \in \mathfrak{R}$ and $a d, c b \in(2,+\infty), e \neq 0$ and $f \neq 0$ then we have

$$
\begin{aligned}
& \lim _{n \rightarrow \infty} x_{2 n-1}=\lim _{n \rightarrow \infty} y_{2 n-1}=0 \\
& \lim _{n \rightarrow \infty} z_{4 n-3}= \begin{cases}0, & c b<a d \\
\infty, & c b>a d \\
\frac{c}{a f}, & c b=a d\end{cases} \\
& \lim _{n \rightarrow \infty} z_{4 n-1}= \begin{cases}0, & c b<a d \\
\infty, & c b>a d \\
f, & c b=a d\end{cases}
\end{aligned}
$$

and

$$
\lim _{n \rightarrow \infty} x_{2 n}=\lim _{n \rightarrow \infty} y_{2 n}=\infty
$$




$$
\begin{array}{r}
\lim _{n \rightarrow \infty} z_{4 n-2}=\left\{\begin{array}{cc}
0, & c b<a d \\
\infty, & c b>a d \\
\frac{d}{b e}, & c b=a d
\end{array}\right. \\
\lim _{n \rightarrow \infty} z_{4 n}= \begin{cases}0, & c b<a d \\
\infty, & c b>a d \\
e, & c b=a d\end{cases}
\end{array}
$$

Proof. The proof is clear from Corollary 3.

\section{Acknowledgements}

We are grateful to the anonymous referees for their valuable suggestions that improved the quality of this study.

\section{References}

[1] A. S. Kurbanli, C. Cinar and I. Yalcinkaya, "On the Behavaior of Positive Solutions of the System of Rational Difference Equations $x_{n+1}=\frac{x_{n-1}}{y_{n} x_{n-1}+1}, \quad y_{n+1}=\frac{y_{n-1}}{x_{n} y_{n-1}+1}$," Mathematical and Computer Modelling, Vol. 53, No. 5-6, 2011, pp. 1261-1267. doi:10.1016/j.mcm.2010.12.009

[2] A. S. Kurbanli, "On the Behavior of Solutions of the System of Rational Difference Equations

$x_{n+1}=\frac{x_{n-1}}{y_{n} x_{n-1}-1}, \quad y_{n+1}=\frac{y_{n-1}}{x_{n} y_{n-1}-1}, "$ World Applied Sciences Journal, 2010, in Press.

[3] A. S. Kurbanli, "On the Behavior of Solutions of the System of Rational Difference Equations

$x_{n+1}=\frac{x_{n-1}}{y_{n} x_{n-1}-1}, \quad y_{n+1}=\frac{y_{n-1}}{x_{n} y_{n-1}-1}, \quad z_{n+1}=\frac{z_{n-1}}{y_{n} z_{n-1}-1}$,

Discrete Dynamics in Nature and Society, 2011, in Press. doi:10.1155/2011/932362

[4] C. Cinar, "On the Solutions of the Difference Equation $x_{n+1}=\frac{x_{n-1}}{-1+a x_{n} x_{n-1}}, "$ Applied Mathematics and Compulation, Vol. 158, 2004, pp. 793-797.

[5] C. Çinar, "On the Positive Solutions of the Difference Equation System $x_{n+1}=\frac{1}{y_{n}}, y_{n+1}=\frac{y_{n}}{x_{n-1} y_{n-1}}, "$ Applied Mathematics and Computation, Vol. 158, 2004, pp. 303-305. doi:10.1016/j.amc.2003.08.073

[6] G. Papaschinopoulos and C. J. Schinas, "On a System of Two Nonlinear Difference Equations," Journal of Mathematical Analysis and Applications, Vol. 219, No. 1998, pp. 415-426. doi:10.1006/jmaa.1997.5829

[7] G. Papaschinopoulos and C. J. Schinas, "On the System of Two Difference Equations," Journal of Mathematical Analysis and Applications, Vol. 273, No. 2, 2002, pp. 294-309. doi:10.1016/S0022-247X(02)00223-8

[8] A. Y. Özban, "On the System of Rational Difference Equations $x_{n}=\frac{a}{y_{n-3}}, \quad \mathrm{y}_{n+1}=\frac{b y_{n-3}}{x_{n-q} y_{n-q}}, "$ Applied Mathematics and Computation, Vol. 188, No. 1, 2007, pp. 833-837. doi:10.1016/j.amc.2006.10.034

[9] D. Clark and M. R. S. Kulenović, "A Coupled System of Rational Difference Equations," Computers \& Mathematics with Applications, Vol. 43, 2002, pp. 49-867.

[10] D. Clark, M. R. S. Kulenović and J. F. Selgrade, "Global Asymptotic Behavior of a Two-Dimensional Diserence Equation Modelling Competition," Nonlinear Analysis, Vol. 52, No. 7, 2003, pp. 1765-1776. doi:10.1016/S0362-546X(02)00294-8

[11] E. Camouzis and G. Papaschinopoulos, "Global Asymptotic Behavior of Positive Solutions on the System of Rational Difference Equations $x_{n+1}=1+\frac{x_{n}}{y_{n-m}}$,

$y_{n+1}=1+\frac{y_{n}}{x_{n-m}}, "$ Applied Mathematics Letters, Vol. 17,

No. 6, 2004, pp. 733-737.

doi:10.1016/S0893-9659(04)90113-9

[12] X. Yang, Y. Liu and S. Bai, "On the System of High Order Rational Difference Equations

$x_{n}=\frac{a}{y_{n-p}}, \quad y_{n}=\frac{b y_{n-p}}{x_{n-q} y_{n-q}}, "$ Applied Mathematics and Computation, Vol. 171, No. 2, 2005, pp. 853-856. doi:10.1016/j.amc.2005.01.092

[13] X. Yang, "On the System of Rational Difference Equations $x_{n}=A+\frac{y_{n-1}}{x_{n-p} y_{n-q}}, y_{n}=A+\frac{x_{n-1}}{x_{n-r} y_{n-s}}$, Journal of Mathematical Analysis and Applications, Vol. 307, No. 1, 2005, pp. 305-311. doi:10.1016/j.jmaa.2004.10.045

[14] M. R. S. Kulenović and Z. Nurkanović, "Global Behavior of a Three-Dimensional Linear Fractional System of Difference Equations," Journal of Mathematical Analysis and Applications, Vol. 310, No. 2, 2005, pp. 673-689.

[15] A. Y. Özban, "On the Positive Solutions of the System of Rational Difference Equations $x_{n+1}=\frac{1}{y_{n-k}}$,

$y_{n+1}=\frac{y_{n}}{x_{n-m} y_{n-m-k}}$," Journal of Mathematical Analysis and Applications, Vol. 323, No. 1, 2006, pp. 26-32. doi:10.1016/j.jmaa.2005.10.031

[16] Y. Zhang, X. Yang, G. M. Megson and D. J. Evans, "On the System of Rational Difference Equations $x_{n}=A+\frac{1}{y_{n-p}}, y_{n}=A+\frac{y_{n-1}}{x_{n-r} y_{n-s}}, "$ Applied Mathematics and Computation, Vol. 176, No. 2, 2006, pp. 403-408. doi:10.1016/j.amc.2005.09.039 
[17] Y. Zhang, X. Yang, D. J. Evans and C. Zhu, "On the Nonlinear Difference Equation System $x_{n+1}=A+\frac{y_{n-m}}{x_{n}}$, $y_{n+1}=A+\frac{x_{n-m}}{y_{n}}, "$ Computers \& Mathematics with Applications, Vol. 53, No. 10, 2007, pp. 1561-1566.

[18] I. Yalcinkaya and C. Cinar, "Global Asymptotic Stability of two nonlinear Difference Equations $z_{n+1}=\frac{t_{n} z_{n-1}+a}{t_{n}+z_{n-1}}$, $t_{n+1}=\frac{Z_{n} t_{n-1}+a}{Z_{n}+t_{n-1}}, "$ Fasciculi Mathematici, Vol. 43, 2010, pp. 171-180.

[19] E. M. Elsayed, "On the Solutions of Higher Order Rational System of Recursive Sequences," Mathematica Balkanica, Vol. 21, No. 3-4, 2008, pp. 287-296.

[20] I. Yalcinkaya, "On the Global Asymptotic Stability of a Second-Order System of Difference Equations," Discrete Dynamics in Nature and Society, 2008, Article ID 860152, 12 Pages.

[21] E. M. Elabbasy, H. EI-Metwally and E. M. Elsayed, "On the Solutions of a Class of Difference Equations Systems," Demonstratio Mathematica, Vol. 41, No. 1, 2008, pp. 109-122.

[22] E. M. Elsayed, "Dynamics of a Recursive Sequence of Higher Order," Communications on Applied Nonlinear
Analysis, Vol. 16, No. 2, 2009, pp. 37-50.

[23] R. Abu-Saris, C. Cinar and I. Yalcinkaya, "On the Asymptotic Stability of $x_{n+1}=\frac{a+x_{n} x_{n-k}}{x_{n}+x_{n-k}}$, Computers \& Mathematics with Applications, Vol. 56, No. 5, 2008, pp. 1172-1175. doi:10.1016/j.camwa.2008.02.028

[24] R. P. Agarwal, W. T. Li and P. Y. H. Pang, "Asymptotic Behavior of a Class of Nonlinear Delay Difference Equations," Journal of Difference Equations and Applications, Vol. 8, 2002, pp. 719-728. doi:10.1080/1023619021000000735

[25] R. P. Agarwal, "Difference Equations and Inequalites," 2nd Editions, Marcel Dekker, New York, 2000.

[26] I. Yalcinkaya, C. Cinar and D. Simsek, "Global Asymptotic Stability of a System of Difference Equations," Applicable Analysis, Vol. 87, No. 6, June 2008, pp. 689699. doi:10.1080/00036810802140657

[27] E. M. Elsayed, "On the Solutions of a Rational System of Difference Equations," Fasciculi Mathematici, Vol. 45 2010, pp. 25-36.

[28] B. Irićanin and S. Stević, "Some Systems of Nonlinear Difference Equations of Higher Order with Periodic Solutions," Dynamics of Continuous, Discrete and Impulsive Systems. Series A Mathematical Analysis, Vol. 13, No. 3-4, 2006, pp. 499-507. 